\title{
Self-Stimulated Capillary Jet
}

\author{
H. González®,$^{1, *}$ J. Arcenegui®,$^{2}$ F.J. García de Bollullos $\odot,{ }^{3}$ J.R. Castrejón-Pita $\odot,{ }^{4}$ and \\ A.A. Castrejón-Pita $\odot^{2, \dagger}$ \\ ${ }^{1}$ Departamento de Física Aplicada III. Escuela Técnica Superior de Ingenieros, Universidad de Sevilla, \\ Camino de los Descubrimientos, Sevilla s/n 41092, Spain \\ ${ }^{2}$ Department of Engineering Science, University of Oxford, Parks Road, Oxford OX1 3Pj, United Kingdom \\ ${ }^{3}$ Departamento de Física Aplicada I. Escuela Politécnica Superior, Universidad de Sevilla, c/ Virgen de África, \\ 7, Sevilla 41011, Spain \\ ${ }^{4}$ School of Engineering and Material Science, Queen Mary University of London, Mile End Road, London E1 4NS, \\ United Kingdom
}

(Received 10 July 2020; revised 10 December 2020; accepted 17 December 2020; published 28 January 2021)

\begin{abstract}
Inspired by Savart's pioneering work, we study the self-stimulated dynamics of a capillary jet. The feedback loop is realized by extracting surface perturbations from a section of the jet itself via a laserphotodiode pair, whose amplified signal drives an electromechanical actuator that, in turn, produces pressure perturbations at the exit chamber. Under specific conditions, this loop establishes phase-locked stimulation regimes that overcome the otherwise random natural breakup. For each laser position along the jet, the gain of the amplifier acts as a selector across a discrete set of observable frequencies. The main observed features are explained by a linear theory that combines the transfer function of each stage in the loop. Our findings are relevant to continuous inkjet technologies for the production of equally sized droplets.
\end{abstract}

DOI: 10.1103/PhysRevApplied.15.014054

\section{INTRODUCTION}

The natural and forced breakup of liquid jets has captured the imagination of scientists and engineers for hundreds of years, from the great and inquisitive mind of Da Vinci, to rigorous mathematicians and experimentalists such as Savart, Magnus, Plateau, Lord Rayleigh, and Bohr [1]. Jet disintegration into a train of droplets is ubiquitous in nature; to mention a few quaint examples, water, blood, and poison jets are used as hunting or defense mechanisms by the archer fish $[2,3]$, the horned lizard, and the bombardier beetle.

Physically, the capillary instability [1] is responsible for the breakup of a liquid jet, and was mathematically described by Rayleigh in 1892. In his model, a harmonic perturbation at the jet exit is advected, leading to a varicose, locally sinusoidal pattern. The Rayleigh-Plateau dispersion relation [4] provides the unstable wavelength range. It also identifies an optimal wavelength with the highest growth rate that, under unforced exit conditions, determines the natural size of the drops after breakup. In practice, a multitude of effects might modify the true value of the optimal instability wavelength, e.g., the velocity relaxation at the exit $[5,6]$, shearing forces with

\footnotetext{
*helio@us.es

†alfonso.castrejon-pita@wadham.ox.ac.uk
}

the surrounding fluid [7,8], gravity [9], and, importantly, the liquid properties (which may be dynamic in nature) $[10,11]$.

From the industrial point of view, continuous inkjet (CIJ) printing can be singled out as the technology where a better understanding of the stimulated breakup of capillary jets may have the most impact. In CIJ printing, a micrometer-sized jet of ink is harmonically stimulated by a piezoelectric or a heating element to induce capillary instabilities that ultimately break the jet into equally spaced droplets. During the last four decades, significant industrial efforts have been dedicated to improving the control over the position, number, size, and directionality of the droplets and their satellites, while also investigating material compatibility needed to adapt the technology to underexplored applications, such as electronics, biotechnology, and microchemistry [12-14]. The complexities that exist in both the inkjet printhead and fluid properties hinder a trustworthy theoretical prediction of the breakup length and the optimal frequency needed to minimize it. Consequently, in industry, the trial and error approach is currently the only one available. Moreover, this process must be performed every time a new ink is developed, which is both resource intensive and time consuming $[13,14]$.

Clearly, industry would benefit from simplifying this procedure. A research and development specialist would like practical answers to the following questions. How do 
we break a jet up at a prescribed distance from the nozzle? Are all the properties of the ink and the stimulation device required? Surprisingly, the key could be hidden in the pioneering experiment of Savart [15] on capillary jets without external perturbations. In order to explain the unexpectedly stable periodical breakup, he identified a feedback mechanism between the downstream droplet splashing and the upstream jet birth, via the supporting structure. Inspired by him, we propose here a phase-locked self-stimulation of a capillary jet through a mechanical actuator and an optical feedback loop to easily determine the optimum stimulation parameters that require a minimum amplitude to induce its breakup at a prescribed position. The experimental realization and theoretical analysis that we present can be easily adapted, not only to other stimulation devices (piezoelectric, thermal, electrical), but also to a variety of feedback methods (image based, charge based, mechanical, among others).

In the following sections we first present the experimental setup and procedure devised for this study, followed by the theoretical treatment utilized to understand this self-stimulated breakup phenomenon. We then present and discuss our combined theoretical and experimental results and offer potential applications in the context of inkjet printing.

\section{EXPERIMENT}

In Fig. 1 we show a sketch of the experimental system and the breakup of a liquid jet due to the capillary instability. The working fluid (here pure deionized water at $21^{\circ} \mathrm{C}$, dynamic viscosity $\mu=1.0 \mathrm{mPa} \mathrm{s}$, surface tension $\gamma=0.072 \mathrm{~N} \mathrm{~m}^{-1}$, and density $\rho=997.6 \mathrm{~kg} / \mathrm{m}^{3}$ ) is

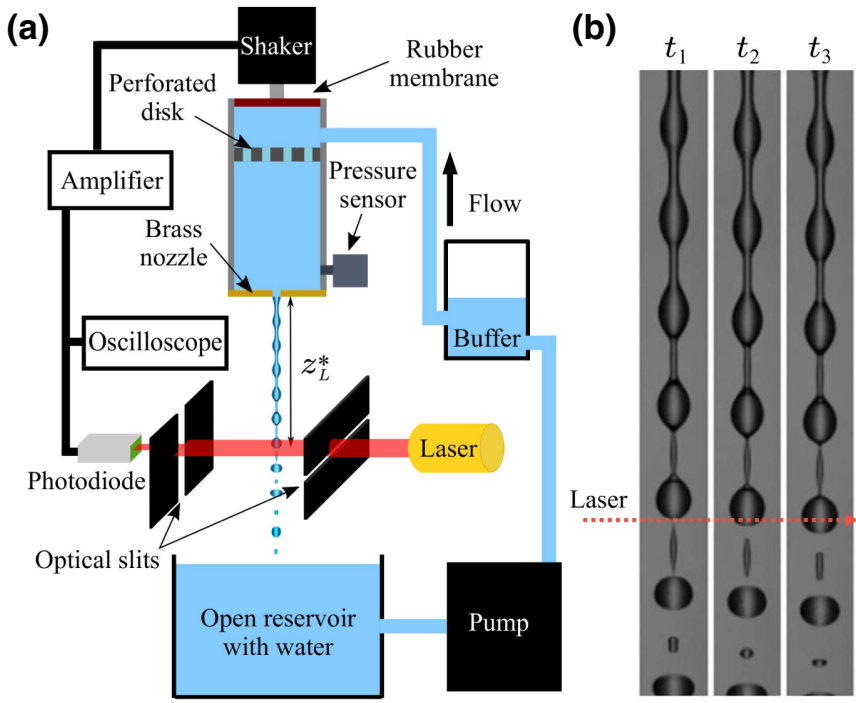

FIG. 1. (a) Sketch of the experimental setup. (b) Snapshots of the jet at times $t_{1}<t_{2}<t_{3}$, with corresponding photodiode output voltages $V\left(t_{1}\right)>V\left(t_{2}\right)>V\left(t_{3}\right)$. pumped from an open reservoir to a pressurized cylindrical ejection chamber. The bottom wall of this chamber forms the nozzle plate with an exit orifice in its center (diameter $1.00 \mathrm{~mm}$ and thickness $0.16 \mathrm{~mm}$ ). The top of the chamber comprises a rubber membrane connected to an electromagnetic actuator for driving pressure perturbations. The flow rate is $Q=1.97 \times 10^{-6} \mathrm{~m}^{3} / \mathrm{s}$ for all the experiments. The jet velocity after relaxation is estimated to be $v_{j}=$ $3.16 \pm 0.02 \mathrm{~m} / \mathrm{s}$, measured by the single-pressure-pulse method [16]. To this end, images are taken with a Phantom Miro-310 high-speed camera coupled to a Tamron SP AF $90 \mathrm{~mm} \mathrm{~F} / 2.8$ Macro 1:1 lens; different frame rates are used in the range from 10000 to $30000 \mathrm{fps}$. After jetting, the liquid is collected by a reservoir, where it is pumped back to the pressurized chamber to close the hydraulic circuit. A $1.0 \mathrm{~mW} 650 \mathrm{~nm}$ laser diode (HERO Laser Module, model PLP6501AR) coupled to a collimating lens and a $50-\mu \mathrm{m}$-thick optical slit generate a horizontal laser sheet (with adjustable width through a second, vertical slit) that locally illuminates the jet. This way, the jet reflects and refracts (but not appreciably absorbs) that light, which is collected by a photodiode aligned to the beam at the other side of the jet. The time-dependent voltage signal from the photodiode (proportional to the incoming light except for the addition of a constant) is then analyzed by a digital oscilloscope (Lecroy HDO4024), electronically amplified, and fed back to the electromechanical actuator. Therefore, the locally varying shape of the jet's surface is ultimately responsible for the stimulation of the jet itself. In Fig. 1(b) we illustrate the relation between the instantaneous jet diameter and the photodiode output voltage at an (adjustable) axial position $z_{L}^{*}$ from the nozzle, as defined in Fig. 1(a). The experiment is set up on an optical table to reduce the effect of the surrounding noise.

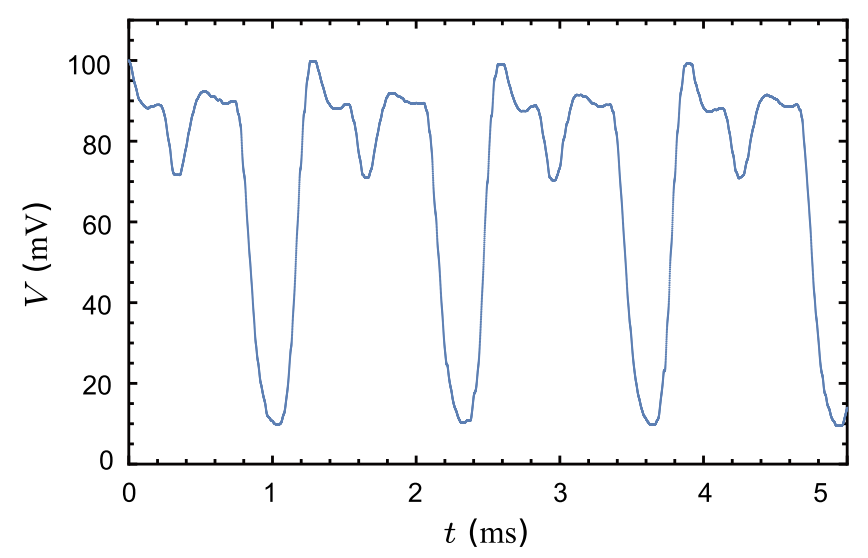

FIG. 2. Output voltage from the photodiode versus time in a typical measurement with the oscilloscope for a stable periodic regime. The zero voltage level is arbitrary. In each period, the absolute minimum comes from the passage of a main drop, while the second largest minimum corresponds to a satellite drop. 
The experimental procedure is as follows: once a position $z_{L}^{*}$ of the laser-photodiode pair is selected along the trajectory of the jet, the electronic gain is increased until a threshold is reached, above which a stable periodic (although nonsinusoidal) signal is displayed by the oscilloscope, as shown in Fig. 2. This is a clear signature of self-stimulation, with a fixed pinch-off point always coincident with the laser position, as confirmed by highspeed-camera monitoring. The oscillation period is then extracted from this signal. By subtly changing the gain, other stable periodic regimes are found. Finally, the position of the laser is changed and the procedure repeated for a large selection of $z_{L}^{*}$.

\section{THEORY}

A theoretical framework is proposed as having a feedback loop comprising four stages: (1) the actuator, (2) the nozzle, (3) the jet, and (4) the photodiode. Each stage has an input and an output quantity coupled via a transfer function (either in the temporal or in the spectral domain). These functions are described in dimensionless form with scales based on the jet radius after relaxation $R_{j}$ for length, the capillary time $t_{c} \equiv\left(\rho R_{j}^{3} / \gamma\right)^{1 / 2}$ for time, $\gamma / R_{j}$ for pressure, and arbitrary units for voltage.

Stage (1): actuator. The electromechanical actuator exerts a force proportional to the driving voltage $V(t)$ producing a pressure perturbation $p(t)$ inside the ejection chamber, which is measured as a voltage signal through a pressure transducer (MEAS EPX-N12-0.35B) located $4 \mathrm{~mm}$ above the nozzle. By sweeping in frequency $v$ within the range of interest, and measuring the pressure response with a lock-in amplifier (Anfatec eLockIn203), or by real-time analysis of both acquired voltage signals, the complex transfer function between Fourier components $A(\omega) \equiv \hat{p}(\omega) / \hat{V}(\omega)=|A| e^{i \phi_{A}}$ is determined, as shown in Fig. 3. The formal dependence on the dimensionless angular frequency $\omega \equiv 2 \pi \nu t_{c}$ is adopted for convenience.

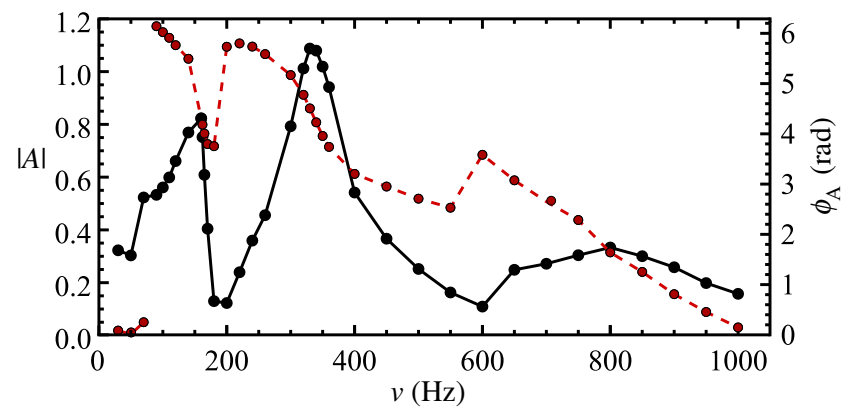

FIG. 3. Measured amplitude (solid line, scale on the left axis in arbitrary units) and phase (dashed line, scale on the right) of the actuator transfer function in terms of the driving frequency. The phase jump at $v=70 \mathrm{~Hz}$ is $2 \pi$, i.e., without physical origin. Experimental errors are smaller than symbols.
Stage (2): nozzle. A transfer function between the harmonic perturbations on the pressure in the chamber and on the jet axial velocity (averaged on a slice) after relaxation was developed in Ref. [16] using a generalized Bernoulli equation. For arbitrary functions of time, this transfer function can be expressed as the ratio of their respective Fourier components, $\hat{p}(\omega)$ and $\hat{w}_{0}(\omega)$, i.e.,

$$
N(\omega) \equiv \hat{w}_{0}(\omega) / \hat{p}(\omega)=\beta /\left(a_{\mathrm{visc}}+i a_{\mathrm{iner}} \omega\right),
$$

where $\beta \equiv v_{j} t_{c} / R_{j}=7.76$ is the dimensionless jet velocity, and $a_{\text {visc }}=2.78$ and $a_{\text {iner }}=0.89$ are dimensionless coefficients, defined in Ref. [16], related to the viscous dissipation and inertia, respectively, inside and near the nozzle.

Stage (3): jet. The capillary instability amplifies the exit perturbations downstream. To account for the weak stretching due to gravity, we replace $v_{j}$ by $v_{m}$, which is the velocity averaged along the intact length of the jet, and $R_{j}$ by $R_{m} \equiv\left(Q / \pi v_{m}\right)^{1 / 2}$ as the new length scale (e.g., $\left.z \equiv z^{*} / R_{m}\right)$. All the dimensionless parameters are accordingly redefined. We make use of the spatial bimodal theory developed in Ref. [17], which considers harmonic perturbations of the form $F(z, t)=1+\operatorname{Re}\left[f(z) e^{-i \omega t}\right]$ on the jet radius and $W(z, t)=\beta+\operatorname{Re}\left[w(z) e^{-i \omega t}\right]$ on the mean axial velocity, and only requires the two downstream capillary modes, $f(z)=f_{d} e^{i k_{d} z}+f_{s} e^{i k_{s} z}$ [and similar expressions for $w(z)]$. Here, $k_{d}$ and $k_{s}$ are complex functions of the real parameter $\omega$, the dimensionless velocity $\beta$, and the Ohnesorge number, $\mathrm{Oh} \equiv \mu /\left(\rho \gamma R_{m}\right)^{1 / 2}$, satisfying a dispersion relation (see Refs. $[18,19]$ for the exact three-dimensional formulation or Ref. [20] for a simpler approximate one-dimensional model). The dominant and subdominant spatial growth rates are given by $-\operatorname{Im}\left[k_{d}\right]$ and $-\operatorname{Im}\left[k_{s}\right]$, respectively. For our pure-impulse conditions, the amplitudes fulfill $f_{d}=-k_{d} k_{s} w_{0} /\left[2 \omega\left(k_{d}-k_{s}\right)\right]=-f_{s}$, where $w_{0}=w(0)$. By interpreting $w_{0}$ as a Fourier component $\hat{w}_{0}(\omega)$ of an arbitrary function of time, the shape reads $f(z, t)=\int_{-\infty}^{\infty} d \omega \hat{w}_{0}(\omega) J(\omega, z) e^{-i \omega t}$ with

$$
J(\omega, z)=\frac{-k_{d} k_{s}}{2 \omega\left(k_{d}-k_{s}\right)}\left(e^{i k_{d} z}-e^{i k_{s} z}\right) .
$$

The jet transfer function $J\left(\omega, z_{L}\right)$ determines the deformation at the laser position for a given velocity perturbation at the exit. As the jet breakup is a fully nonlinear phenomenon, our linear formulation is an approximation that will be discussed later.

Stage (4): photodiode. The output voltage provided by the photodiode is proportional to the instantaneous jet shape deformation at position $z_{L}$ [see Fig. 1(b)], except for an offset associated with the average received light. Consequently, the laser-photodiode pair and the signal amplifier are incorporated into the formulation by $V(t+$ $\left.t_{0}\right)=-G f\left(z_{L}, t\right)+V_{\text {off }}$, with $V(t)$ being the output from 
the amplifier, $V_{\text {off }}$ the offset, $G$ (hereinafter called the gain) a positive-defined parameter combining a fixed gain from the photodiode and a controllable gain from the amplifier, and $t_{0}$ the total electronic delay (negligible in our experiments). Note from Fig. 1(b) that a positive deformation implies a reduction in the light captured by the photodiode.

With these four transfer functions, we find (in the spectral domain) $\hat{V}(\omega) e^{-i \omega t_{0}}=-G A(\omega) N(\omega) J\left(\omega, z_{L}\right) \hat{V}(\omega)$, which is only satisfied by a monochromatic function $\hat{V}(\omega) \propto \delta\left(\omega-\omega_{n}\right)$, with $\omega_{n}$ being a discrete set of frequencies given by the condition

$$
e^{-i \omega_{n} t_{0}}=-G_{n} A\left(\omega_{n}\right) N\left(\omega_{n}\right) J\left(\omega_{n}, z_{L}\right)
$$

for some positive $G_{n}$. The imaginary part of Eq. (3) determines the admissible $\omega_{n}$. The criterion is of a phaselocking type. To show this, $k_{d}$ and $k_{s}$ are expressed in terms of the temporal growth rates $\alpha_{d}$ and $\alpha_{s}$ (assuming that $\beta \gg 1)$ through $k_{d} \simeq k-i \alpha_{d} / \beta$ and $k_{s} \simeq k-$ $i \alpha_{s} / \beta$ with $k \equiv \omega / \beta$ [17]. Then, $J(\omega, z) \simeq J(\beta k, z) \equiv$ $-i(k / 2) e^{i k z}\left(e^{\alpha_{d} z / \beta}-e^{\alpha_{s} z / \beta}\right) /\left(\alpha_{d}-\alpha_{s}\right)$. As $\alpha_{d}$ and $\alpha_{s}$ are real, the imaginary part of Eq. (3) simplifies to

$$
\operatorname{Im}\left[A\left(\omega_{n}\right) N\left(\omega_{n}\right) i e^{i k_{n} z} e^{i \omega_{n} t_{0}}\right]=0
$$

with $k_{n}=\omega_{n} / \beta$. If we could unrealistically, and only for the sake of the theoretical analysis, neglect the effects of the delay $t_{0}$, the nozzle $N(\omega)$, and the actuator $A(\omega)$, we would obtain $k_{n} z_{L}-\pi / 2=2 \pi n$ for all integers $n$ fulfilling $0<k_{n}<1$ (unstable range) and $G_{n}>0$, which clearly reveals the phase-locking nature of condition (4). Each positive $G_{n}$ verifying the real part of Eq. (3) determines the gain that holds the periodic regime having the frequency $\omega_{n}$. In physical terms, the gain acts as a frequency selector.

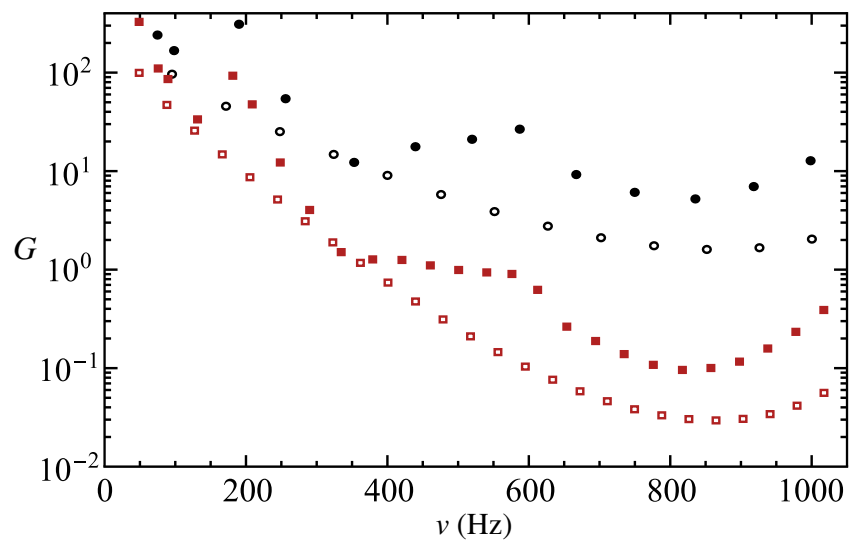

FIG. 4. Discrete sets of gains, $G_{n}$ (in arbitrary units), allowing periodic regimes, versus frequencies at the two representative laser positions, $z_{L}^{*}=43.00 \mathrm{~mm}$ (circles) and $z_{L}^{*}=85.23 \mathrm{~mm}$ (squares). Parameters are $\beta=7.9$ for circles and 8.0 for squares, and $\mathrm{Oh}=0.0056$ for both. Empty symbols correspond to gains calculated without the actuator influence.
In Fig. 4 we show the discrete spectra for two significantly different laser positions. A threshold gain $G_{\min }\left(z_{L}^{*}\right)$ must be overcome in order to achieve a periodic selfstimulated regime. The actuator strongly influences the spectra, as illustrated in Fig. 4, both including and excluding the effect of the actuator transfer function. Note that (i) frequencies are not equispaced, (ii) $G_{\min }$ is much lower for the largest $z_{L}^{*}$ due to a greater spatial amplification, and (iii) the spectrum is denser for the same $z_{L}$ because the highest $n$ fulfilling $k_{n}<1$ increases roughly linearly with $z_{L}$.

Gravity stretches and accelerates the jet, thus producing a gradual variation of any wave number and its associated growth rates along the jet [9]. An improved phase-locking condition (4) can be found by abandoning averages along the intact length, like $R_{m}$, and substituting $k z_{L}$ with an integrated phase, i.e., $\int_{0}^{z_{L}^{*}} d z^{*} k_{\text {loc }}^{*}\left(z^{*}\right)$ (asterisks denote dimensional quantities), where the local wave number is taken as $k_{\mathrm{loc}}^{*}=\omega^{*} / v^{*}\left(z^{*}\right)$. This integral comes from the usual WKB approximation [9]. For the basic flow, we have $R_{j}^{2} v_{j}=R^{*}\left(z^{*}\right)^{2} v^{*}\left(z^{*}\right)$, leading to $k_{\mathrm{loc}}^{*}=$ $\omega^{*} R^{*}\left(z^{*}\right)^{2} /\left(v_{j} R_{j}^{2}\right)$. Assuming free-fall stretching, $R^{*}=$ $R_{j}(1+2 z / \mathrm{Fr})^{-1 / 4}$ [9], where $z=z^{*} / R_{j}$ and $\mathrm{Fr} \equiv v_{j}^{2} /\left(g R_{j}\right)$ is the Froude number $\left(2.32 \times 10^{3}\right.$ in our experiments $)$, we arrive at

$$
\int_{0}^{z_{L}^{*}} d z^{*} k_{\mathrm{loc}}^{*}\left(z^{*}\right)=k_{j} \operatorname{Fr}\left(\sqrt{1+\frac{2 z_{L}}{\mathrm{Fr}}}-1\right)
$$

with $k_{j} \equiv \omega^{*} R_{j} / v_{j}$. As Fr $\rightarrow \infty$, Eq. (5) becomes $k_{j} z_{L}$.

\section{RESULTS}

In Fig. 5 we compare, in terms of $z_{L}^{*}$, measured frequencies (crosses) with our two theoretical discrete spectra. Circles come from Eq. (3); their gray level graphically quantifies the gain relative to its lowest value for that position $z_{L}^{*}$. The lines, generated by Eqs. (4) and (5), better account for gravity effects and, indeed, they substantially improve the agreement with our experiments. The best agreement is found for the laser positions that are far from the nozzle and for frequencies $v \simeq 800 \mathrm{~Hz}$, i.e., around the maximum of the Rayleigh curve $(k \simeq 0.69)$. These frequencies also correspond to the lowest gains needed to obtain locked behavior (highest gray levels).

The prediction of a discrete set of frequency states for each $z_{L}^{*}$ is confirmed in most runs with $z_{L}^{*}>60 \mathrm{~mm}$ (up to five frequencies for $z_{L}^{*}=84 \mathrm{~mm}$ ). In the experiments, these states are explored by slightly changing the gain, sometimes by tapping. It is noteworthy that Savart selected different frequencies with a single bow strike from a violin [15], but returned to the "natural frequency" by tapping the supporting structure. As seen in Fig. 4, metastable states are expected from our analysis because several frequencies have similar gains near the local minimum at $800 \mathrm{~Hz}$. Interestingly, for laser positions around $z_{L}^{*} \simeq 60 \mathrm{~mm}$, the system 


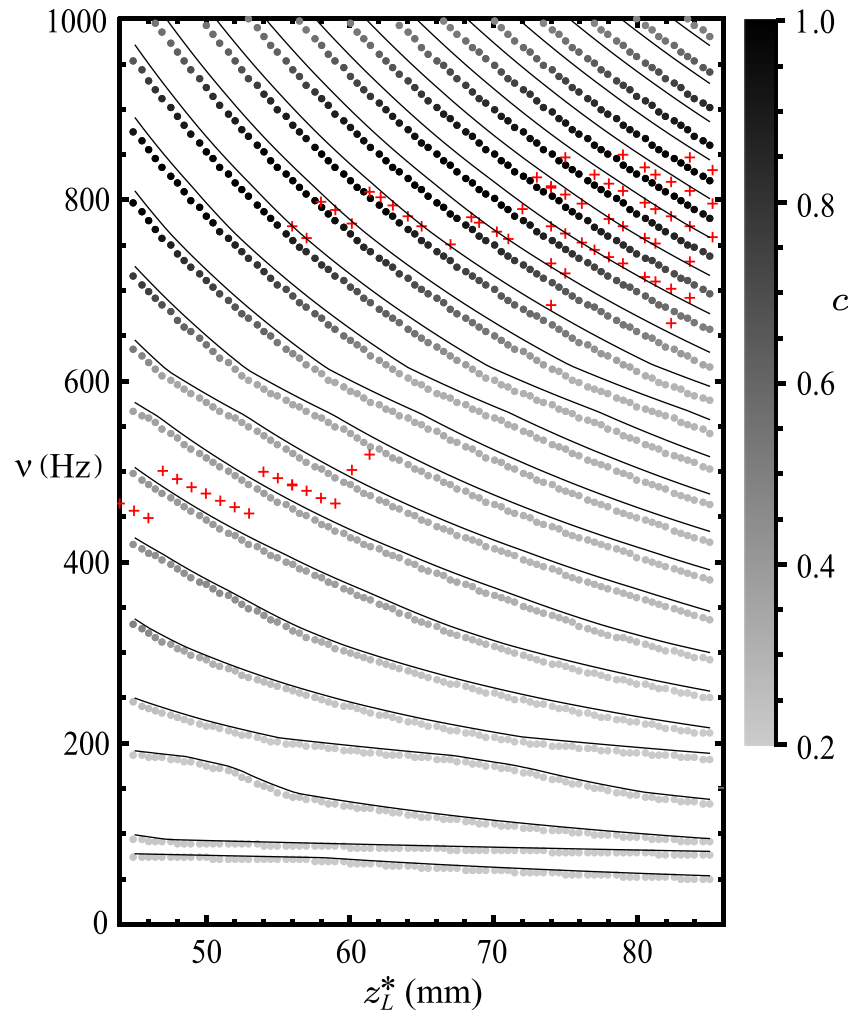

FIG. 5. Discrete spectrum for each laser position $z_{L}^{*}$ without gravity corrections from Eq. (3) (circles) and with gravity corrections from Eqs. (4) and (5) (lines). The gray level $c$ of each circle (scale on the right) shows its required gain according to $c\left(G_{n}, z_{L}^{*}\right)=0.2+0.8 G_{\min }\left(z_{L}^{*}\right) / G_{n}$ [black for $c=1$, i.e., $G_{n}=$ $G_{\min }\left(z_{L}^{*}\right)$; clearest gray for $c=0.2$ as $\left.G_{n} \rightarrow \infty\right]$. Crosses are the experimental data.

also selects a frequency in the range $v \simeq 450-500 \mathrm{~Hz}$, which becomes the only one observed for $z_{L}^{*}<56 \mathrm{~mm}$.

Aimed at justifying our linear approximation, an auxiliary experiment has explored the effects of limiting the laser sheet width by narrowing the width of the vertical slit [see Fig. 1(a)]. Thereby, a threshold is imposed to the amplitude of the jet deformation monitored by the photodiode. Reducing the laser width leads to a sinusoidlike signal (weaker deformation) and a breakup displaced downstream. However, the signal period remains constant within our experimental accuracy, thus demonstrating that the linear map of Fig. 5 can predict the selected frequencies despite the breakup being a nonlinear process.

\section{DISCUSSION AND CONCLUDING REMARKS}

The physical picture of the process sustaining the periodic regime is as follows. The signal driving the actuator remains the same after a feedback cycle (no jump in both phase and amplitude) if condition (3) is satisfied. While our predictions hold for monochromatic regimes, the photodiode obviously acquires not only the fundamental frequency but the whole combination of harmonics that describe the breakup shape. Incidentally, the relationship between the diameter of the thread and the photodiode's output voltage is only approximate since some light, transmitted through the center of both the jet and the drops, as well as that diffracted by their edges, is also being detected. A more accurate and linear relationship between the instantaneous voltage and the corresponding jet diameter can be achieved for opaque working fluids (such as inks) and by using noncoherent light sources [21]. Whatever their origin (nonlinear or optical), the harmonics, introduced into the exit conditions in the next cycle, become exponentially negligible, provided they are outside the unstable wave number region [22]. Downstream, they reappear due to the nonlinear jet dynamics, thus producing the same breakup. Accordingly, only the fundamental Fourier component is relevant, in agreement with the result of our auxiliary experiment.

As far as the initial perturbation, estimated through $f_{0}=\exp \left[-\alpha_{d}(k) z_{L} / \beta\right]$, is small enough, our linear theory should hold, since the amplitude remains small during most of the jet life [23]. However, for short enough jets $\left(z_{L}^{*}\right.$ small), the linear theory is expected to be less accurate. For instance, the initial perturbation estimate at $z_{L}^{*}=45 \mathrm{~mm}$ is $f_{0}=0.13$, certainly not small. This explains the disagreement at $z_{L}^{*}<60 \mathrm{~mm}$ in Fig. 5. Finally, the transition from 800 to $500 \mathrm{~Hz}$ could be caused by (i) the actuator performance (see Fig. 3), and (ii) the instability of the second harmonic (recall that $k<0.5$, so $2 k$ is within the unstable range). We note, in this latter case, that nonmonochromatic states are not treatable by the linear theory.

Regarding applications, this study has the potential to greatly benefit CIJ printing technologies. The selfstimulation allows the determination of the optimal frequency and amplitude that one would need to drive an actuator with, in order to achieve jet breakup at a desired length. The steps are: laser-photodiode pair placement, oscilloscope signal recording, laser-photodiode pair removal, and oscilloscope signal use as the stimulationdriving signal. This signal is optimal as the setup tunes itself to the frequency that needs the minimum stimulation amplitude. Moreover, this method only requires a single experiment, without prior information about the working fluid or the hydraulic system.

\section{ACKNOWLEDGMENTS}

This work is supported by the Project PGC2018099217-B-I00, funded by ERDF and the Spanish Research Agency Ministerio de Ciencia e Innovación, the EPSRC-UK Starting Grant EP/P024173/1, and an Equipment Award from the Department of Engineering Science, Oxford. A.A.C.-P. is further supported by the Royal Society through a University Research Fellowship URF/R/180016 and an Enhancement Grant RGF/EA/181002. 
[1] J. Eggers and E. Villermaux, Physics of liquid jets, Rep. Prog. Phys. 71, 1 (2008).

[2] A. Vailati, L. Zinnato, and R. Cerbino, How archer fish achieve a powerful impact: Hydrodynamic instability of a pulsed jet in toxotes jaculatrix, PLoS ONE 7, 1 (2012).

[3] P. Gerullis and S. Schuster, Archerfish actively control the hydrodynamics of their jets, Curr. Biol. 24, 2156 (2014).

[4] L. Rayleigh, On the instability of a cylinder of viscous liquid under capillary forces, Philos. Mag. 34, 145 (1892).

[5] A. Sevilla, The effect of viscous relaxation on the spatiotemporal stability of capillary jets, J. Fluid Mech. 684, 204 (2011).

[6] B. Lopez, A. Soucemarianadin, and P. Attané, Break-up of continuous liquid jets: Effect of nozzle geometry, J. Imag. Sci. Tech. 43, 145 (1999).

[7] A. Kalaaji, B. Lopez, P. Attané, and A. Soucemarianadin, Breakup length of forced liquid jets, Phys. Fluids 15, 2469 (2003).

[8] J. M. Gordillo and M. Pérez-Saborid, Transient effects in the signaling problem, Phys. Fluids 14, 4329 (2002).

[9] S. Senchenko and T. Bohr, Shape and stability of a viscous thread, Phys. Rev. E 71, 056301 (2005).

[10] M. Ronay and T. B. Benjamin, Determination of the dynamic surface tension of liquids from the instability of excited capillary jets and from the oscillation frequency of drops issued from such jets, Proc. R. Soc. Lon. Ser.-A 361, 181 (1978).

[11] O. A. Basaran, H. Gao, and P. P. Bhat, Nonstandard inkjets, Annu. Rev. Fluid Mech. 45, 85 (2013).

[12] O. A. Basaran, Small-scale free surface flows with breakup: Drop formation and emerging applications, AIChE J. 48, 1842 (2002).
[13] S. Hoath, Fundamentals of Inkjet Printing: The Science of Inkjet and Droplets (Wiley, Weinheim, Germany, 2015).

[14] B. Derby, Inkjet printing of functional and structural materials: Fluid property requirements, feature stability, and resolution, Annu. Rev. Mater. Res. 40, 395 (2010).

[15] F. Savart, Mémoire sur la constitution des veines liquides lancées par des orifices circulaires en mince paroi, Annal. Chim. 53, 337 (1833).

[16] F. J. García, H. González, J. R. Castrejón-Pita, and A. A. Castrejón-Pita, The breakup length of harmonically stimulated capillary jets, Appl. Phys. Lett. 105, 094104 (2014).

[17] H. González, P. A. Vazquez, F. J. García, and J. Guerrero, Minimal formulation of the linear spatial analysis of capillary jets: Validity of the two-mode approach, Phys. Rev. Fluids 3, 044802 (2018).

[18] S. J. Leib and M. E. Goldstein, Convective and absolute instability of a viscous jet, Phys. Fluids 29, 952 (1986).

[19] J. Guerrero, H. González, and F. J. García, Spatial modes of capillary jets, with application to surface stimulation, J. Fluid Mech. 702, 354 (2012).

[20] J. Guerrero, H. González, and F. J. García, Spatial modes in one-dimensional models for capillary jets, Phys. Rev. E 93, 033102 (2016).

[21] H. González and F. J. García, The measurement of growth rates in capillary jets, J. Fluid Mech. 619, 179 (2009).

[22] P. A. Torpey, A nonlinear theory for describing the propagation of disturbances on a capillary jet, Phys. Fluids A 1, 661 (1989).

[23] F. J. García and H. González, Normal-mode linear analysis and initial conditions of capillary jets, J. Fluid Mech. 602, 81 (2008). 